\title{
Evaluation de la contamination des éléments traces métalliques dans les sédiments de la rivière N'zi, Côte d'Ivoire
}

\author{
Ahbeauriet Ahmed OUATTARA ${ }^{1,2^{*}}$, Naminata SANGARE ${ }^{\mathbf{1}}$, Koffi Pierre dit Adama \\ $\mathrm{N}^{\prime}$ GORAN ${ }^{3}$, Koffi Marcellin YAO $^{2}$, Albert TROKOUREY ${ }^{1}$ et Thomas DIACO ${ }^{1}$ \\ ${ }^{1}$ Laboratoire de Constitution et Réaction de la Matière, Université Félix Houphouët Boigny, Abidjan, \\ Côte d'Ivoire. \\ ${ }^{2}$ Centre de Recherches Océanologiques (CRO), Abidjan, Côte d'Ivoire. \\ ${ }^{3}$ UFR Sciences Biologiques de l'Université Peleforo Gon Coulibaly, BP 1328 Korhogo, Côte d'Ivoire. \\ *Auteur correspondant; E-mail: ahbeauriet@gmail.com
}

\begin{tabular}{lll}
\hline Received: 20-06-2021 & Accepted: 07-10-2021 & Published: 30-10-2021 \\
\hline
\end{tabular}

\section{RESUME}

Les éléments traces métalliques s'accumulent dans les systèmes aquatiques et peuvent dégrader la qualité des eaux et présenter une menace pour la santé humaine et la faune. Cependant peu d'étude existe sur la concentration totale des métaux dans les cours d'eau ivoiriens impactées par les activités agricoles. Pour contribuer à la mise en place d'une base de données, nous avons étudié la distribution et le niveau de contamination du $\mathrm{Cu}, \mathrm{Hg}, \mathrm{Pb}, \mathrm{Ni}$ et $\mathrm{Zn}$ dans les eaux et les sédiments de la rivière N'zi. La concentration des éléments traces métalliques $(\mathrm{Cu}, \mathrm{Hg}, \mathrm{Pb}, \mathrm{Ni}$ et $\mathrm{Zn})$ ont été mesurées dans les sédiments de la rivière N'zi pour évaluer le niveau de pollution. Le degré et les sources de contamination ont été déterminés par le calcul des indices de pollution métallique : facteur de contamination (FC), indice de géoaccumulation (Igeo), facteur d'enrichissement (FE) indice global de pollution (PLI). Les valeurs de Igeo pour le Cd et le Cu ont été supérieures à 0 et les valeurs de $\mathrm{FC}$ pour le $\mathrm{Cd}, \mathrm{Cu}$ et le $\mathrm{Hg}$ ont été supérieures à 1 dans la station de Moofoué, ce qui suggère que les sédiments de Moofoué sont modérément contaminés par ces éléments. Les valeurs de PLI ont montré que tous les sédiments sont non pollués. Les valeurs de FE pour le cuivre ont excédé 1,5 ce qui dénote une origine anthropique pour le $\mathrm{Cd}$ et le $\mathrm{Cu}$.

(C) 2021 International Formulae Group. All rights reserved.

Mots clés: Métaux traces, sédiments, indices de contamination, rivière.

\section{Assessment of trace metals contamination in sediments of N'zi River, Côte d'Ivoire}

\begin{abstract}
Traces metal elements accumulate in aquatic systems and can degrade water quality and pose a threat to human health and wildlife. However, few studies exist on the total concentration of metals in ivorian rivers impacted by agricultural activities. To help set up a database, we studied the distribution and level of contamination of $\mathrm{Cu}, \mathrm{Hg}, \mathrm{Pb}, \mathrm{Ni}$ and $\mathrm{Zn}$ in the waters and sediments of the N'zi River. The concentrations of the trace metals $(\mathrm{Cu}, \mathrm{Hg}, \mathrm{Ni}, \mathrm{Pb}$ and $\mathrm{Zn})$ were measured for the surface sediments of the N'zi River, Côte d'Ivoire, to evaluate the level of contamination. Sediment pollution assessment was carried out using contamination factor
\end{abstract}


(CF), pollution load index (PLI), geoaccumulation index (Igeo) and enrichment factor (EF). The Igeo value for $\mathrm{Cd}$ and $\mathrm{Cu}$ were $>0$ and the $\mathrm{CF}$ values for $\mathrm{Cd}, \mathrm{Cu}$ and $\mathrm{Hg}$ were $>1$ in sediments of the Moofoué station, which denotes a moderate contamination in these metals. The PLI indicated that all stations were no polluted. The EF value for $\mathrm{Cd}$ and $\mathrm{Cu}$ were $>1.5$ in the sediments, suggesting anthropogenic impact on the metal levels in the river. The concentrations of $\mathrm{Cd}$ and $\mathrm{Cu}$ are likely to result in harmful effects on sediment-dwelling organisms which are expected to occur frequently based on the comparison with sediment quality guidelines.

(C) 2021 International Formulae Group. All rights reserved.

Keywords: Trace metals, sediment, index risk, river.

\section{INTRODUCTION}

Ces dernières décennies, la pollution de l'environnement par les métaux traces est devenue un sujet de préoccupation mondiale, en raison de leur résistance à la biodégradation, leur toxicité et leur capacité à s'incorporer dans la chaîne alimentaire (Koaussi et al., 2015 ; Ouattara et al., 2018 ; Kinimo et al., 2018 ; Ouattara et al., 2020). Les métaux traces sont introduits dans l'environnement à travers des phénomènes naturels et des activités anthropiques telles que les activités minières, agricoles, industrielles et les effluents domestiques (N'guessan et al., 2009 ; Du et al., 2013). La pollution de l'environnement par les métaux traces augmente avec l'intensification des activités anthropiques et l'urbanisation accrue (Soro et al., 2009). L'intensification de la pollution des milieux aquatiques par les métaux traces peut avoir des effets néfastes sur l'homme et son environnement.

Une fois dans le milieu aquatique, les métaux traces se répartissent entre la phase aqueuse, les sédiments et les organismes aquatiques (Ouattara et al., 2020 ; Togbe et al., 2019). Les métaux traces se lient principalement aux particules sédimentaires et peuvent s'accumuler dans les sédiments. Les sédiments représentent donc des réservoirs pour les métaux traces dans l'environnement. Lorsque des changements physico-chimiques ( $\mathrm{pH}$, salinité, matières en suspension, turbidité) se produisent, les métaux traces piégés dans les sédiments sont susceptibles d'être rélargués dans la colonne d'eau (Zhang et al., 2014) et contaminer les eaux et les organismes aquatiques (Aduayi-akue et al., 2014 ; Yehouenou et al., 2010). Ainsi, les sédiments sont utilisés comme des indicateurs du niveau de pollution de l'environnement. L'évaluation du niveau de contamination des sédiments par les métaux traces permet de comprendre l'impact des activités anthropiques sur les milieux aquatiques.

Dans les zones rurales, l'usage massif des fertilisants et des pesticides représentent les principales sources de contamination en éléments trace métallique pour les milieux aquatiques (Ouattara et al., 2018 ; Zhang et al., 2014). Les eaux du bassin versant du N'zi ne sont pas en reste. En effet, le bassin versant du N'zi-Bandama est une zone à forte production agricole. L'étude de la contamination métallique des eaux de ce bassin est d'une importance capitale, car elles sont utilisées à des fins multiples (boisson, lessive...) par les populations rurales sans traitement préalable.

En Côte d'Ivoire, peu d'études ont été menées sur les métaux dans les rivières en zone agricole. Notre travail avait pour objectif d'étudier la distribution et le niveau de contamination des métaux $\mathrm{Cu}, \mathrm{Ni}, \mathrm{Zn}, \mathrm{Pb}$ et $\mathrm{Hg}$ dans les eaux et les sédiments de la rivière N'zi. Pour atteindre cet objectif, l'étude portait sur la détermination du degré d'accumulation des métaux à partir des indices de contamination dans les sédiments.

\section{MATERIEL ET METHODES Sites d'échantillonnage}

Le N'zi est le principal affluent de rive gauche du fleuve Bandama dont la confluence se trouve à 10 kilomètres au nord de Tiassalé. La zone de la rivière étudiée est située au sud du bassin versant dans la région de l'AgnebyTiassa, précisément dans le département de Tiassalé. Cette zone est comprise entre le village de Moofoué et une plantation industrielle de banane douce de la société SCB 
(Figure 1). Les prélèvements ont été effectués sur cinq stations le long de la zone d'étude représentées par la Figure 1.

Liste des stations :

- Station 1: Moofoué Latitude $6^{\circ} 07^{\prime} 05.9^{\prime \prime N}$

Longitude $4^{\circ} 44^{\prime} 44.1^{\prime \prime} \mathrm{W}$

- Station 2: Apiamoh Latitude $6^{\circ} 06^{\prime} 01.3^{\prime \prime} \mathrm{N}$

Longitude $4^{\circ} 45^{\prime} 54.4^{\prime \prime} \mathrm{W}$

- Station 3: Djibi Latitude $6^{\circ} 05^{\prime} 23.6^{\prime \prime} \mathrm{N}$ Longitude $4^{\circ} 45^{\prime} 81.2^{\prime \prime} \mathrm{W}$

- Station 4 : N'zianouan Latitude $6^{\circ} 00^{\prime} 12.5^{\prime \prime} \mathrm{N}$

Longitude 449'21.0"W

- Station 5: SCB Latitude $5^{\circ} 59^{\prime} 42.8^{\prime \prime} \mathrm{N}$ Longitude $4^{\circ} 49^{\prime} 56.6 " \mathrm{~W}$.

\section{Echantillonnage}

L'échantillonnage des sédiments de surface a été réalisé au moyen d'une petite benne de type Van-Veen, suivant les méthodes de références pour les études de la pollution marine (UNEP, 2007). Les sédiments ont été conservés dans des flacons en polyéthylène, puis placés à l'obscurité dans une glacière (4 $\left.{ }^{\circ} \mathrm{C}\right)$. Au laboratoire, les sédiments ont été séchés à l'étuve $\left(40^{\circ} \mathrm{C}\right)$ pendant 72 heures, les sédiments ont été conservés dans des flacons en plastique, avec un ruban en plastique autour du dispositif de fermeture afin de les protéger contre l'humidité, puis stockés dans une armoire à l'obscurité $\left(20^{\circ} \mathrm{C}\right)$ pour analyse.

\section{Dosage des métaux traces.}

Pour la détermination de la concentration totale des métaux $\mathrm{Fe}, \mathrm{Hg}, \mathrm{Cu}, \mathrm{Ni}$, $\mathrm{Pb}$ et $\mathrm{Zn}$, les sédiments ont été digérés par décomposition totale selon les méthodes de références pour les études de la pollution marine (UNEP, 2007).

Le principe de la méthode de digestion est basé sur la décomposition des sédiments par l'acide hydrofluorique (HF) en combinaison avec de l'eau régale $\left(\mathrm{HNO}_{3}: \mathrm{HCl} ; 1: 3, \mathrm{v} / \mathrm{v}\right)$ à chaud. L'utilisation de HF est essentielle parce que cet acide est le seul acide à dissoudre complètement les treillis silicatés et tous les métaux (Loring et Rantala, 1992; Chakraborty, 2012).

Les métaux ont été dosés par Spectrométrie d'Absorption Atomique (SAA) de type Varian AA 20. La limite de détection pour l'analyse de métaux a été de $0,02 \mu \mathrm{g} / \mathrm{L}$ pour le $\mathrm{Cu}$ et le $\mathrm{Ni}, 0,06 \mu \mathrm{g} / \mathrm{L}$ pour le plomb et $0,05 \mu \mathrm{g} / \mathrm{L}$ pour le $\mathrm{Zn}$.

Le mercure a été analysé par spectroscopie d'absorption atomique en utilisant un analyseur direct de mercure type AMA-254 (Altech,). La limite de détection pour l'analyse du mercure total était de $1 \mathrm{ng} / \mathrm{g}$.

\section{Contrôle qualité et méthodes statistiques}

L'assurance de la qualité et le contrôle de la qualité ont été évalués en utilisant des blancs et des matériaux de référence standard. La précision de la méthode de détermination a été systématiquement et régulièrement examiné avec des matériaux de référence standard (GSF). Chaque échantillon a été analysé en double ou en triple. La précision de la mesure, calculée en multipliant l'écart-type par 3, a varié entre $1 \%$ et $6 \%$ ). Elle a été inférieure à $1 \%$ pour le mercure total.

Tous les traitements statistiques: moyenne, les représentations graphiques ont été effectuées à l'aide du logiciel Microsoft Excel 2007.

\section{Calcul des indices d'accumulation métallique}

L'Indice de géoaccumulation (Igéo) donne des informations sur le niveau d'accumulation des éléments métalliques dans le sédiment. Il est évalué à partir de la relation ci-dessous:

$$
I_{\text {géo }}=\log 2\left(\frac{C_{n}}{1,5 \times B_{n}}\right)
$$

Où:

$\mathrm{C}_{\mathrm{n}}$ : concentration du métal $\mathrm{n}$ dans le sédiment; $\mathrm{B}_{\mathrm{n}}$ : valeur préindustrielle pour le métal $\mathrm{n} ; 1,5$ : constance prenant en compte les fluctuations naturelles de la teneur d'une substance donnée dans un environnement ainsi que les fluctuations anthropiques.

Les valeurs d'Igéo permettent de définir sept classes de niveau de contamination rassemblées dans le Tableau 1 (Müller, 1981)

Le FC permet de connaître le niveau de contamination métallique des sédiments. Il est calculé à partir de la relation:

$$
\mathbf{F C}=\frac{\mathrm{C}_{\text {métal }}}{\mathrm{C}_{\text {bruit de fond métal }}}
$$


Avec:

$\mathrm{C}_{\text {métal }}$ : concentration du métal dans le sédiment; $\mathrm{C}_{\text {bruit de fond métal }}$ : valeur du bruit de fond géochimique pour le métal.

Les différents niveaux de contamination selon les valeurs de FC sont regroupés dans le Tableau 2.

Le FE a été proposé pour discriminer les apports anthropiques des sources naturelles. Le facteur d'enrichissement est calculé à partir de la relation suivante:

$$
\mathbf{F E}=\frac{([\mathrm{X}] /[\mathrm{R}]) \text { échantillon }}{([\mathrm{X}] /[\mathrm{R}]) \text { matériel de référence }}
$$

Avec:

$\mathrm{X}$ : élément étudié; R: élément normalisant.

Dans cette étude le fer a été choisi comme élément normalisant. Les valeurs de FE comprises entre 0,5 et 1,5 indiquent une origine naturelle des métaux, alors que celles supérieures à 1,5 sont attribuées à des apports anthropiques (Zhang et al., 204).
L'indice de la charge de pollution (PLI: Pollution Load Index en Anglais), permet d'estimer le degré de contamination global des sédiments de la zone étudiée, à partir de la concentration totale de tous les métaux étudiés. L'équation suivante a été développée par Tomlinson et al. (1980) pour le calcul de l'indice de charge polluant (PLI):

$\mathbf{P L I}=\sqrt[\mathbf{n}]{\mathrm{FC}_{\mathbf{1}} \times \mathrm{FC}_{\mathbf{2}} \times \mathrm{FC}_{\mathbf{3}} \times \ldots \times \mathrm{FC}_{\mathrm{n}}}$

Avec: $\mathrm{FC}_{\mathrm{i}}$ : facteur de contamination du métal i.

L'indice de pollution métallique donne des informations cumulées sur la pollution métallique dans les sédiments. Pour PLI $=0$, il n'y a pas de détérioration; pour PLI $=1$, seuls les niveaux de référence des polluants sont présents, et la valeur de PLI > 1 indique une détérioration progressive de l'estuaire.

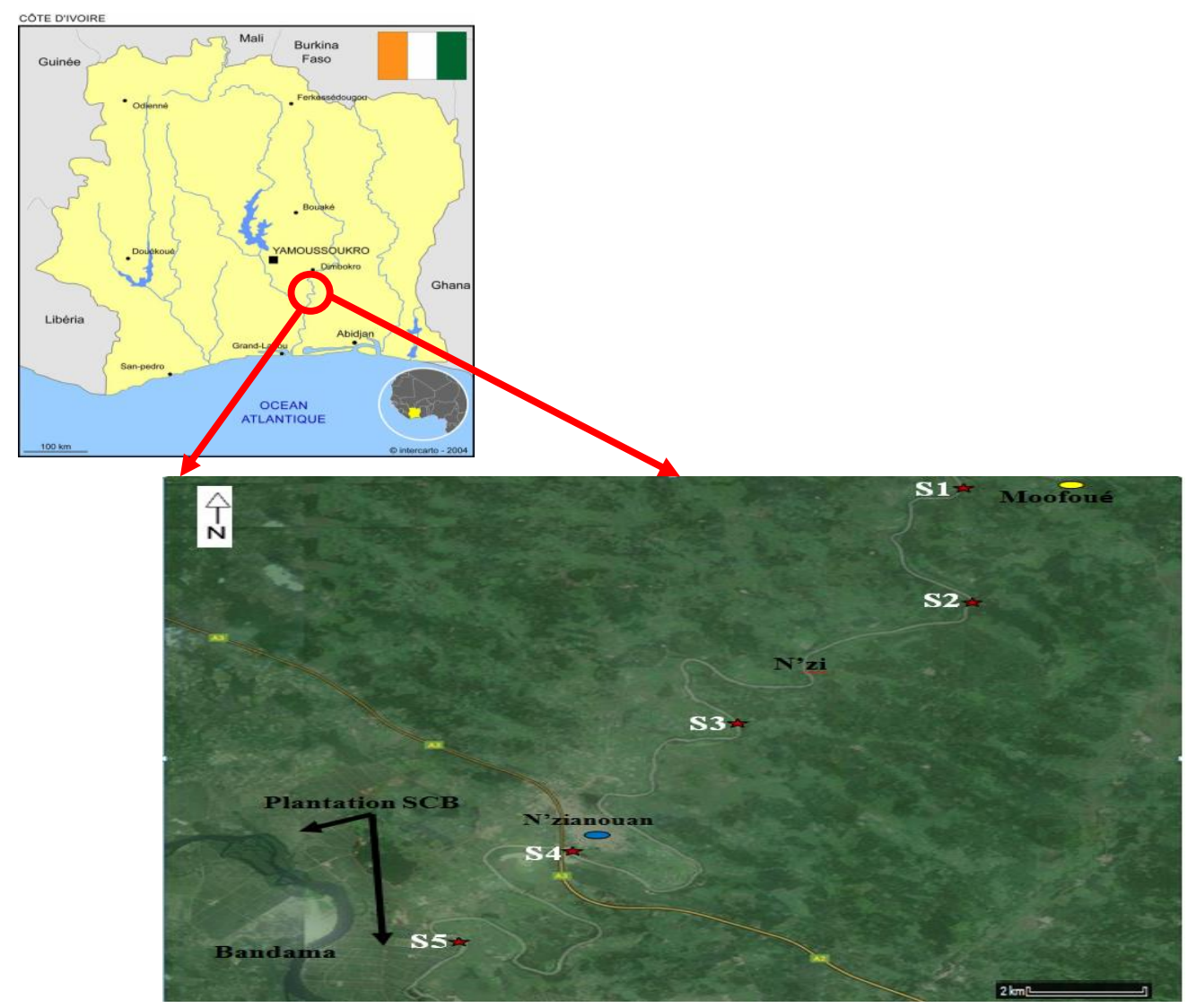

Figure 1: Localisation des sites d'échantillonnage sur le N'zi. 
Tableau 1: Différentes classes selon Müller (1981).

\begin{tabular}{lll}
\hline Classe & Valeurs & Niveau de contamination \\
\hline 0 & Igéo $<0$ & Non contaminé \\
1 & $0<$ Igéo $<1$ & De non contaminé à modérément contaminé \\
2 & $1<$ Igéo $<2$ & Modérément contaminé \\
3 & $2<$ Igéo $<3$ & De modérément contaminé à sévèrement contaminé \\
4 & $3<$ Igéo $<4$ & Sévèrement contaminé \\
5 & $4<$ I géo $<5$ & De sévèrement contaminé à extrêmement contaminé \\
6 & $5<$ Igéo & Extrêmement contaminé \\
\hline
\end{tabular}

Tableau 2: Niveau de contamination suivant les valeurs de FC.

\begin{tabular}{ll}
\hline Valeur de FC & Contamination \\
\hline $\mathrm{FC}<1$ & Faible contamination \\
$1 \leq \mathrm{FC}<3$ & Contamination modéré \\
$3 \leq \mathrm{FC}<6$ & Contamination considérable \\
$\mathrm{FC} \geq 6$ & Très forte contamination \\
\hline
\end{tabular}

\section{RESULTATS}

\section{Distribution des métaux $\mathrm{Cu}, \mathrm{Hg}, \mathrm{Zn}, \mathrm{Pb}$ et Ni dans les sédiments}

Les concentrations des métaux cuivre, mercure, zinc, plomb et nickel dans les sédiments des stations sont représentées dans le Tableau 3.

Les concentrations en métaux traces ont été comprises entre nd et $38,83 \mu \mathrm{g} / \mathrm{g}$. Les moyennes ont été de $14,73 \pm 14,54 \mu \mathrm{g} / \mathrm{g}$ pour le $\mathrm{Cu} ; 0,65 \pm 0,57 \mu \mathrm{g} / \mathrm{g}$ pour le $\mathrm{Pb} ; 0,49 \pm 0,05$ $\mu \mathrm{g} / \mathrm{g}$ pour le $\mathrm{Cd} ; 0,03 \pm 0,001 \mu \mathrm{g} / \mathrm{g}$ pour le $\mathrm{Hg}$; $1,66 \pm 0,91$ pour le $\mathrm{Ni}$ et $3,16 \pm 2,03$ pour le Zinc. La concentration la plus élevée a été le $\mathrm{Cu}$ dans la station de Moofoué. L'ordre de contamination des sédiments s'est établi comme suit : Moofoué $>$ SCB $>$ N'zianouan > Apiamoh > Djibi.

Afin d'évaluer le degré de contamination des sédiments de la rivière N'zi, les indices de contamination ont été calculés.

\section{Degré de contamination}

Les valeurs du FC des métaux dans les sédiments des stations sont representées par la
Figure 2. Les échantillons de sédiments prélevés dans les stations de Apiamoh, Djibi et SCB sont faiblement contaminés par tous les métaux étudiés indiqués par des valeurs de FC comprises entre 0,02 et 0,84 . Pour les sédiments de Moofoué les valeurs du FC obtenues avec le $\mathrm{Cu}, \mathrm{Hg}$ et le $\mathrm{Cd}$ ont été de 2,71, 1,14 et 5,46 jugeant ces sédiments modérément contaminés en $\mathrm{Cu}$ et en $\mathrm{Hg}$ et considérablement contaminés en $\mathrm{Cd}$. Tandis qu'à N'zianouan, les sédiments sont faiblement contaminés $(0,16 \leq \mathrm{FC} \leq 0,81)$ en $\mathrm{Cu}, \mathrm{Ni}, \mathrm{Pb}$ et $\mathrm{Zn}$ et considérablement contaminés en $\mathrm{Cd}$.

Les valeurs de l'indice de géoaccumulation $(-11,93 \leq$ Igéo $\leq 1,79)$ calculées pour tous les métaux sont représentées par la Figure 3. Les valeurs ont montré que les sédiments de Moofoué et de N'zianouan sont modérément contaminés en $\mathrm{Cd}$, les sédiments de Moofoué sont de non contaminés à modérément contaminés en $\mathrm{Cu}$.

Les FE calculés sont indiqués dans le Tableau 4. Les résultats montrent que pour les valeurs de $\mathrm{FE}$ pour le $\mathrm{Cd}$ excèdent largement 1,5 dans les sédiments de Moofoué et 
N'zianouan, ainsi pour le $\mathrm{Cu}$ dans les sédiments de Moofoué. Cela suggère un enrichissement anthropique par le $\mathrm{Cd}$ et le $\mathrm{Cu}$. Les sédiments des différentes stations seraient naturellement enrichis en $\mathrm{Zn}, \mathrm{Ni}, \mathrm{Pb}$ et en $\mathrm{Hg}$.

Les variations de l'indice global PLI sont représentées par la Figure 4.
Les résultats indiquent qu'il n'y a pas de détérioration progressive des sédiments des stations par les métaux étudiés. En effet, les valeurs de PLI sont comprises entre $0,00 \leq$ PLI $\leq 0,32$.

Tableau 3 : Concentration en métaux traces $(\mu \mathrm{g} / \mathrm{g})$ dans les sédiments.

\begin{tabular}{lllllll}
\hline & $\mathbf{C u}$ & $\mathbf{P b}$ & $\mathbf{C d}$ & $\mathbf{H g}$ & $\mathbf{N i}$ & $\mathbf{Z n}$ \\
\hline Moofoué & 38,83 & 0,30 & 0,53 & 0,064 & 3,12 & 1,16 \\
Apiamoh & 0,05 & 0,2 & nd & nd & 1,07 & 6,08 \\
Djibi & nd & nd & nd & nd & 0,52 & 2,3 \\
N'zianouan & 11,62 & 0,48 & 0,44 & 0,002 & 1,43 & 1,20 \\
SCB & 8,42 & 1,63 & nd & 0,009 & 2,18 & 5,04 \\
\hline UCC & 14,3 & 17 & 0,102 & 0,056 & 18,6 & 52 \\
\hline
\end{tabular}

*nd : non déterminé

*UCC : valeurs mondiales préindustrielles

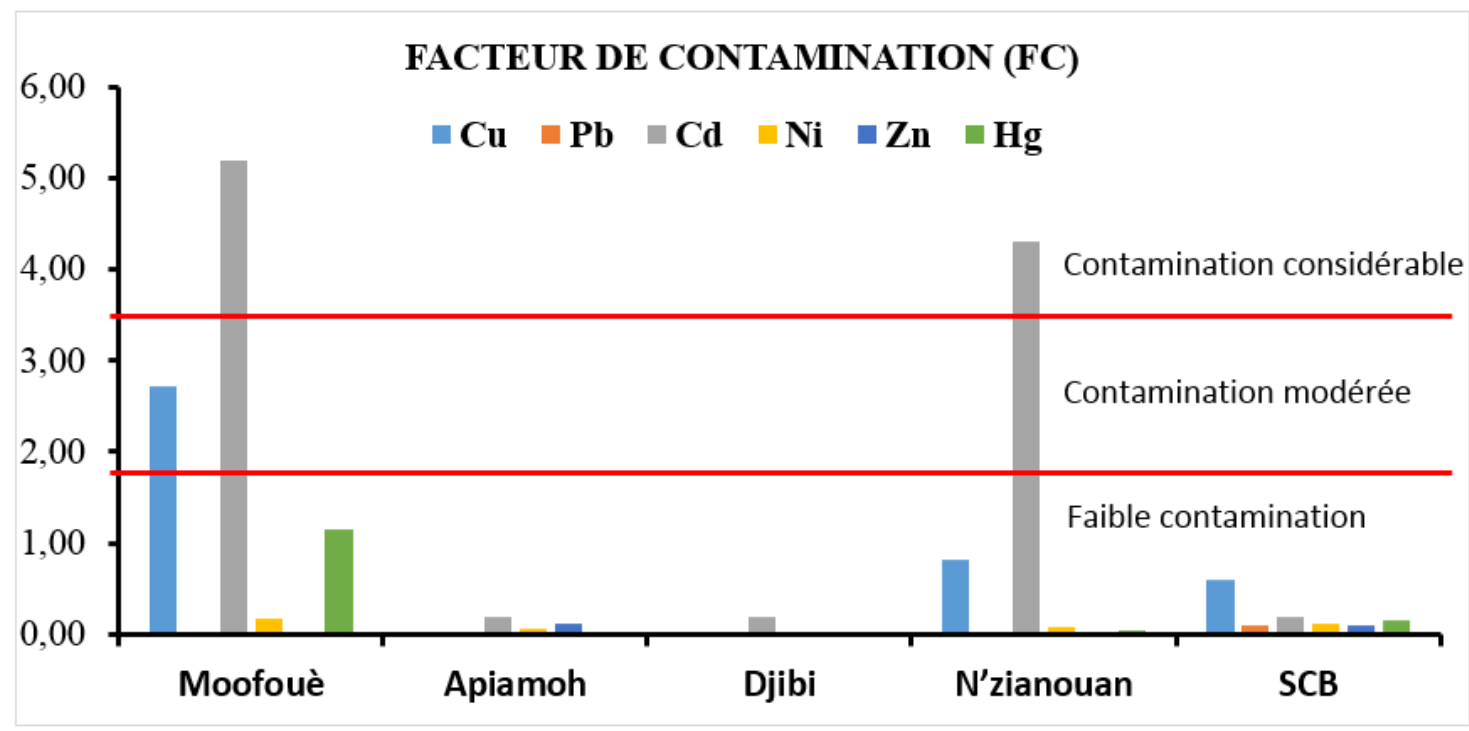

Figure 2: Variations du Facteur de Contamination dans les sédiments. 


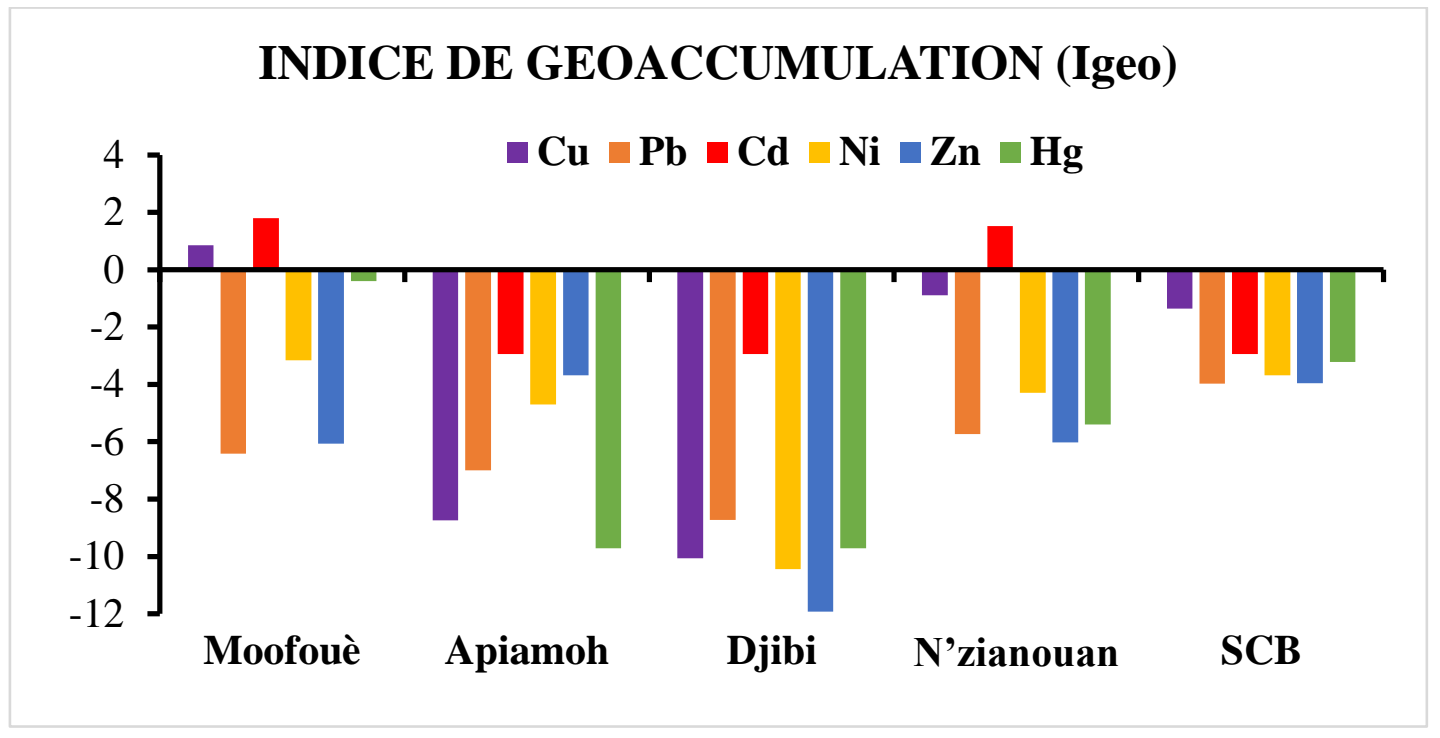

Figure 3: Variations de l'Indice de géoaccumulation dans les sédiments.

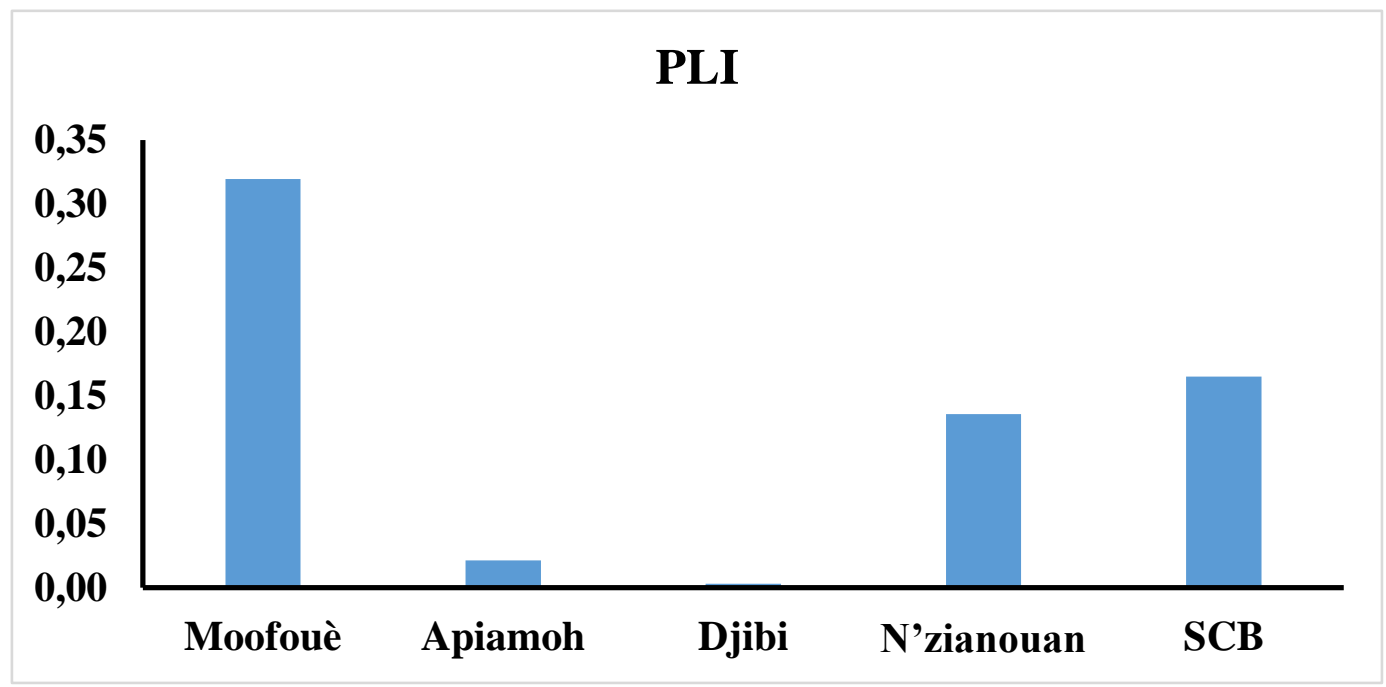

Figure 4: Variations de l'indice de la charge de pollution métallique dans les sédiments.

Tableau 4: Valeur du facteur d'enrichissement (FE) dans les sédiments.

\begin{tabular}{ccccccc}
\hline Station & $\mathbf{C u}$ & $\mathbf{P b}$ & $\mathbf{C d}$ & $\mathbf{N i}$ & $\mathbf{Z n}$ & $\mathbf{H g}$ \\
\hline Moofoué & 2,48 & 0,02 & 4,74 & 0,15 & 0,02 & 1,04 \\
Apiamoh & 0 & 0,01 & 0,17 & 0,05 & 0,1 & 0 \\
Djibi & 0 & 0 & 0,18 & 0 & 0 & 0 \\
N'zianouan & 0,73 & 0,03 & 3,9 & 0,07 & 0,02 & 0,03 \\
SCB & 0,57 & 0,09 & 0,19 & 0,11 & 0,09 & 0,16 \\
\hline
\end{tabular}




\section{DISCUSSION}

Les résultats ont montré une forte teneur des sédiments en $\mathrm{Cu}, \mathrm{Cd}$ et $\mathrm{Hg}$ à la station de Moofoué largement supérieure à la valeur préindustrielle de la haute croûte continentale (Wedepohl, 1995). Cette forte teneur suggère une contamination d'origine anthropique (Harguinteguya et al., 2013 ; Paramasivam et al., 2015). Cette accumulation peut être due aux activités locales telles que l'usage des herbicides et des engrais, aux activités domestiques et à l'usage du charbon de bois. En effet, les engrais et herbicides sont utilisés couramment dans cette zone pour traiter les pépinières d'hévéa. La concentration plus élevée du $\mathrm{Hg}$ obtenue dans les sédiments de Moofoué pourrait s'expliquer par les activités de charbon qui sont courantes dans cette zone. Un échantillonnage couvrant une plus grande surface permettra de mieux confirmer cette suggestion. Par contre, les concentrations moyennes en $\mathrm{Ni}, \mathrm{Pb}$ et $\mathrm{Zn}$ ont été inférieures aux valeurs mondiales préindustrielles UCC indiquant que la source majeure de ces métaux est d'origine naturelle.

Les indices de contaminations ont montré un fort apport anthropique pour le Cd et le $\mathrm{Cu}$ dans les sédiments des stations de Moofoué et de N'zianouan. Ces stations sont des zones à la fois agricoles et urbaines. Les métaux traces dans les sédiments de ces stations sont d'origine agricole et urbaine d'où cette forte contamination des sédiments. Tandis que les autres stations sont principalement agricoles.

\section{Conclusion}

Dans cette étude, les niveaux de contamination en cadmium, cuivre, plomb, nickel, zinc et mercure des sédiments de surface de la rivière N'zi ont été évalués. Les résultats ont montré une forte contamination des sédiments de Moofoué et de N'zianouan en $\mathrm{Cd}$ et en $\mathrm{Cu}$ montrant une contamination par les activités anthropiques en $\mathrm{Cu}$ et en $\mathrm{Hg}$. Le calcul de l'indice de charge de pollution (PLI) a montré qu'il n'y a pas de détérioration progressive de la qualité des sédiments du N'zi. Cependant, le développement des activités économiques dans cette zone pourrait impacter davantage la qualité des eaux du fleuve. Par conséquent, une collecte continue et/ou périodique de données sur la qualité des eaux s'avère essentielle pour préserver la santé des populations et l'écosystème.

\section{CONFLIT D'INTERETS}

Les auteurs déclarent n'avoir aucun conflit d'intérêts.

\section{CONTRIBUTION DES AUTEURS}

La contribution des auteurs à la réalisation des travaux de laboratoire, à la rédaction et aux différentes corrections s'est établie comme suit : AAO a été l'instigateur et le rédacteur principal de cet article et a effectué les travaux de collecte et de laboratoire; NS a contribué à la rédaction du manuscrit et à l'analyse des données; KPdAN a aidé au traitement des données ; KMY et TD ont conçu et supervisé le travail. AT a contribué par ses critiques avisées et ses conseils.

\section{REMERCIEMENTS}

Les auteurs expriment leur gratitude au Directeur du Centre de Recherches Océanologiques (CRO) pour son soutien et ses encouragements et à l'ensemble des partenaires impliqués dans toutes les opérations afférentes à la collecte et aux traitements des échantillons.

\section{REFERENCES}

Aduayi-Akue AA, Gnandi K. 2014. Evaluation de la pollution par les métaux lourds des sols et de la variété locale du maïs Zea mays dans la zone de traitement des phosphates de Kpémé (Sud du Togo). Int. J. Biol. Chem. Sci., 8(5): 2347-2355.

Chakraborty P. 2012. Speciation of Co, Ni and $\mathrm{Cu}$ in the coastal and estuarine sediments: 
Some fundamental characteristics. Journal of Geochemical Exploration, 115: 13-23. DOI: 10.1016/j.gexplo.2012.01.008.

Du Y, Gao B, Zhou H, Ju X, Hao, Yin S. 2013. Health Risk Assessment of Heavy Metals in Road Dusts in Urban Parks of Beijing, China. Procedia Environmental Sciences, 18: 299-309. DOI: 10.1016/j.proenv.2013.04.039.

Harguinteguya CA, Schreiberb R, Pignataa ML. 2013. Myriophyllumaquaticum as a biomonitor of water heavy metal input related to agricultural activities in the Xanaes River (Córdoba, Argentina). Ecological Indicators, 27: 8-16. DOI: http://dx.doi.org/10.1016/j.ecolind.2012. 11.018 .

Kinimo KC, Yao, KM, Marcotte S, Kouassi NLB, Trokourey A. 2018. Distribution trends and ecological risks of arsenic and trace metals in wetland sediments around gold mining activities in central-southern and southeastern Côte d'Ivoire. Journal of Geochemical Exploration, 190: 265-280. DOI: 10.1016/j.gexplo.2018.03.013.

Kouassi NLB, Yao, KM, Trokourey A, Soro B. 2015. Distribution, Sources, and Possible Adverse Biological Effects of Trace Metals in Surface Sediments of a Tropical Estuary. Environmental Forensics, 16: 96-108.

DOI:

10.1080/15275922.2014.991433.

Loring DH, Rantala RTT. 1992. Manuel for the geochemical analyses of marine sediments and suspended particulate matter. Earth Sciences Reviews, 32(4): 235-283. DOI: 10.1016/00128252(92)90001-A.

Müller G. 1981. Die Schwermetallbelastung der sediment des Neckars und seiner Nebenflusse: eine Bestandsaufnahme. Chemical Zeitung, 105: 157-164.

N'guessan YM, Probst JL, Bur T, Probst A. 2009. Trace elements in stream bed sediments from agricultural catchments (Gascogne region, S-W France): Where do they come from? Science of The Total Environment, 407(8): 2939-2952. DOI: 10.1016/j.scitotenv.2008.12.047.

Ouattara AA, Yao KM, Kinimo KC, Trokourey A. 2020. Assessment and bioaccumulation of arsenic and trace metals in two commercial fish species collected from three rivers of Côte d'Ivoire and health risks. Microchemical Journal, 154: $104604 . \quad$ DOI: https://doi.org/10.1016/j.microc.2020.10 4604.

Ouattara AA, Yao KM, Soro MP, Diaco T, Trokourey A. 2018. Arsenic and Trace Metals in Three West African rivers: Concentrations, Partitioning, and Distribution in Particle-Size Fractions. Archives of Environmental Contamination and Toxicology, 75(3): 449-463. DOI: 10.1007/s00244-0180543-9.

Paramasivam K. Ramasamy V, Suresh G. 2015. Impact of sediment characteristics on the heavy metal concentration and their ecological risk level of surface sediments of Vaigairiver, Tamilnadu, India. Spectrochimica Acta Part A: Molecular and Biomolecular Spectroscopy, 137: 397-407. DOI: http://dx.doi.org/10.1016/j.saa.2014.08.0 56.

Soro G, Metongo BS, Soro N, Ahoussi EK, Kouamé FK, Zade SGP, Soro T. 2009. Métaux lourds ( $\mathrm{Cu}, \mathrm{Cr}, \mathrm{Mn}$ et $\mathrm{Zn}$ ) dans les sédiments de surface d'une lagune tropicale africaine : cas de la lagune Ebrie (Côte d'Ivoire). Int. J. Biol. Chem. Sci., 3(6): 1408-1427.

Togbe AMO, Kouame KV, Yao KM, Ouattara AA, Tidou AS, Atsé BC. 2019. Évaluation de la contamination des eaux de la lagune Ebrié (Zones IV et V), Côte d'Ivoire en arsenic, plomb et cadmium: 
variations spatio-temporelles et risques sanitaires. Int. J. Biol. Chem. Sci., 13(2): 1162-1179.

Tomlinson DL, Wilson JG, Harriis CR, Jeffry DW. 1980. Problem in assessment of heavy levels in estuaries and the formation of pollution index. Helgoländer Meeresuntersuchungen, 33(1-4) : 566-575.

UNEP. 2007. Manuel d'échantillonnage et d'analyse des sédiments UNEP (DEPI) / MED WG.321 / Inf 4. United Nations Environment Program, p 26.

Wedepohl HK. 1995. The composition of the continental crust. Geochimica et Cosmochimica Acta, 59 (7): 1217-1232.
Yehouenou EAP, Soton A, Azocli D, Acakpo H, Boco M, Fourn L, Houinsa D, Keke JC, Fayomi B. 2010. Contamination du sol, de l'eau et des produits maraîchers par des substances toxiques et des métaux lourds sur le site de Houéyiho (Cotonou) en République du Bénin. Int. J. Biol. Chem. Sci., 4(6): 2160-2168.

Zhang C, Yu ZG, Zeng GM, Jiang M, Yang ZZ, Cui F, Zhu MY, Shen L, Hu L. 2014. Effects of sediment geochemical properties on heavy metal bioavailability. Environment International, 73: 270-281. DOI : 10.1016/j.envint.2014.08.010. 